\title{
Discrete event simulation - a tool to support the design of complex production and logistic processes; its application in underground mine design
}

\author{
K Quan Amec Foster Wheeler, Canada \\ G King Amec Foster Wheeler, Australia \\ T Schrimpf Amec Foster Wheeler, Australia
}

\begin{abstract}
Computer simulation modelling has been applied to a wide range of mining, mineral processing and transportation projects. These models have become valuable, as decision support tools, during the planning and design phases of any mining project along the entire value chain from orebody to customer. The significant capital costs associated with such projects, and the undesirable consequences of inopportune decisions, make computer modelling a 'must-do' activity in design optimisation.
\end{abstract}

Mines are complex systems of mobile and fixed equipment operated by humans, each with their own operating rules, schedules, productivity rates, and maintenance requirements. The overall performance of the system depends on many factors and it is the efficiency with which all of the unit operations within the system interact that often determines whether or not the system is optimised or balanced.

Traditional methods of analysis often use averages, which do not account for the 'peaks and valleys' in system performance. A computer simulation model makes it possible to predict the performance of a complex system by mimicking the variability and randomness in its operation, which are more often than not the causes of interruptions in system performance. Using a simulation model to guide our decisions, we can move step-by-step toward a balanced system design; one which minimises capital investment, operating costs, inventory requirements, and opportunity costs of idle equipment.

This paper presents some background on the advantages of using computer simulation modelling with some examples of Amec Foster Wheeler's practice in its application on underground mining projects.

\section{$1 \quad$ Introduction - what is simulation?}

Simulation is the process of designing a computer model of a real system based on mathematical logic. The model steps forward in simulated time taking into account the randomness and chronological sequencing of events observed on real systems. The goal is to mimic the real system with sufficient accuracy to use the model to make projections of real system behaviour under experimental conditions. These projections permit inferences to be drawn about systems without building them (if they are proposed); without disturbing them (if they are operating); and without destroying them (if the goal is to determine their strength). Different design concepts and alternate operating procedures can be evaluated for existing and/or proposed systems.

A discrete-event simulation models the operation of a system as a sequence of distinct events in time where each event marks a change of state in the system. This contrasts with continuous simulation in which the simulation continuously tracks the state of system over time.

Computer simulation modelling can be an integral part of the planning, design and operational analyses of underground and surface mines, process plants and transportation systems. 


\section{$2 \quad$ Why use simulation?}

At its simplest, underground mine planning involves coming up with the optimum layout for the underground workings that get at most of the ore, at the lowest cost, and with the greatest safety (Priyadarshi 2012).

In recent years, the mining industry has faced fierce competition and markets that peak and dip. Understanding an operation and the factors that influence it can mean the difference between profit and loss. Simulation approaches are particularly well suited to improving understanding of and diagnosing issues in complex systems. Key reasons to simulate complex mining systems are to optimise production and to mitigate risk.

Other reasons to simulate mining systems are:

- Identify system bottlenecks.

- Quantify production rates and capacities for equipment and facilities.

- Test alternatives without shutdowns or loss of production.

- Determine system focus and strategic direction.

- Allocate resources.

- Provide decision support for engineering.

- Develop operating procedures and philosophies.

- Train operators.

- Mitigate risk.

- Save capital.

In underground mines, the movement of ore from the working face to the surface is a complex logistics network comprised of mobile and fixed equipment, often operated by human crews. Mine production depends on the inter-activity of these unit operations.

\section{Static versus dynamic modelling}

More often than not, production capacity is estimated using static methods, e.g. linear calculations using a spreadsheet. These are well-suited for preliminary estimates but they typically only consider average values for mining rates, ore handling capacities, mobile equipment cycle times, equipment downtimes etc. 'Upset' events such as machine breakdowns, traffic congestion, work stoppages for crews, etc. are usually included as line items and don't include the impact of variable durations on the overall system.

Dynamic models, e.g. discrete-event simulation models, take into account these factors and can give a more detailed picture of how an operation will behave under various conditions (Quan \& Durston 2003). Even though the overall, long-term average performance of the system may be the same as that predicted by the static model, a dynamic model provides more detailed statistical output such that the performance of a system, when it deviates from steady state, can be quantified.

Static models:

- Good for initial analysis.

- Not time based.

- Estimates steady-state performance.

- Limited ability to capture variability and inter-activity of unit operations. 
Dynamic models:

- More complex.

- Incorporate variability and detailed historic data.

- More closely mimics the system being modelled.

- Provides detailed information about the behaviour of the system.

Underground mining production is a start and stop process. For example, at the beginning of a shift there is a delay in production to allow crews to travel to the work places. Once production starts, it may pause for any number of planned and unplanned events, e.g. machine movement, crew break, unplanned failure of a piece of equipment etc. The net result is 'peaks and valleys' in instantaneous mine output. Typically, these changes in mine production rates can be buffered (smoothed out) by installing surge inventory capacity within the mine (orepasses, bins etc.). However, the exact number of these storages, where they are located, and how big they need to be, can drive the capital cost of the project up or down.

A simple example of the difference between the outputs of a static model compared to those of a dynamic model can be illustrated in equipment availability and its impact on an ore handling system.

Consider a gyratory crusher with an installed capacity of 2,000 t/h and an equipment availability of $95 \%$. This implies that this piece of equipment is in operating condition $95 \%$ of the time. In a static analysis, this figure is typically used as a factor against the installed nameplate capacity of the equipment:

$$
2,000 \frac{t}{h} x 95 \%=1,900 \frac{t}{h}
$$

This figure of $1,900 \mathrm{t} / \mathrm{h}$ would be used as the steady state average performance rate for the crusher.

However, equipment availability of $95 \%$ does not provide the whole picture of equipment downtime and its impact on the overall system. The following combinations of time to repair (TTR) and time between failures (TBF) all equal 95\% availability:

- 5-day outage every 100 days.

- 5-hour outage every 100 hours.

- 5-minute outage every 100 minutes.

The frequency and duration of the outages each have a profoundly different effect on the overall system. Short, more frequent outages would likely not impact downstream operations as much as a long, albeit less frequent outage. A crusher that stops operating due to a blockage that can be cleared in a few minutes would not interrupt the mine in the same way a broken crusher shaft that requires a day or two to be replaced.

In reality, the downtimes on the crusher would be a combination of short, more frequent downtimes and long, less frequent downtimes - each having a different impact on the system.

A model of the crusher was built with $95 \%$ availability using the following inputs:

- Assume 5-hour outage every 100 hours on average.

- Mean time between failures (MTBF): 100 hours (use a lognormal distribution).

- Mean time to repair (MTTR): 5 hours (use an exponential distribution). 
The model was run for 100,000 simulated hours (approximately 1,000 failure events), which resulted in the following outputs:

- Average TBF: $94.97 \mathrm{~h}$.

- Minimum TBF: $33.69 \mathrm{~h}$; maximum TBF: $228.59 \mathrm{~h}$.

- Average TTR: $4.82 \mathrm{~h}$.

- Minimum TTR: $0.01 \mathrm{~h}$; maximum TTR: $32.75 \mathrm{~h}$.

These results are shown graphically in Figure 1.

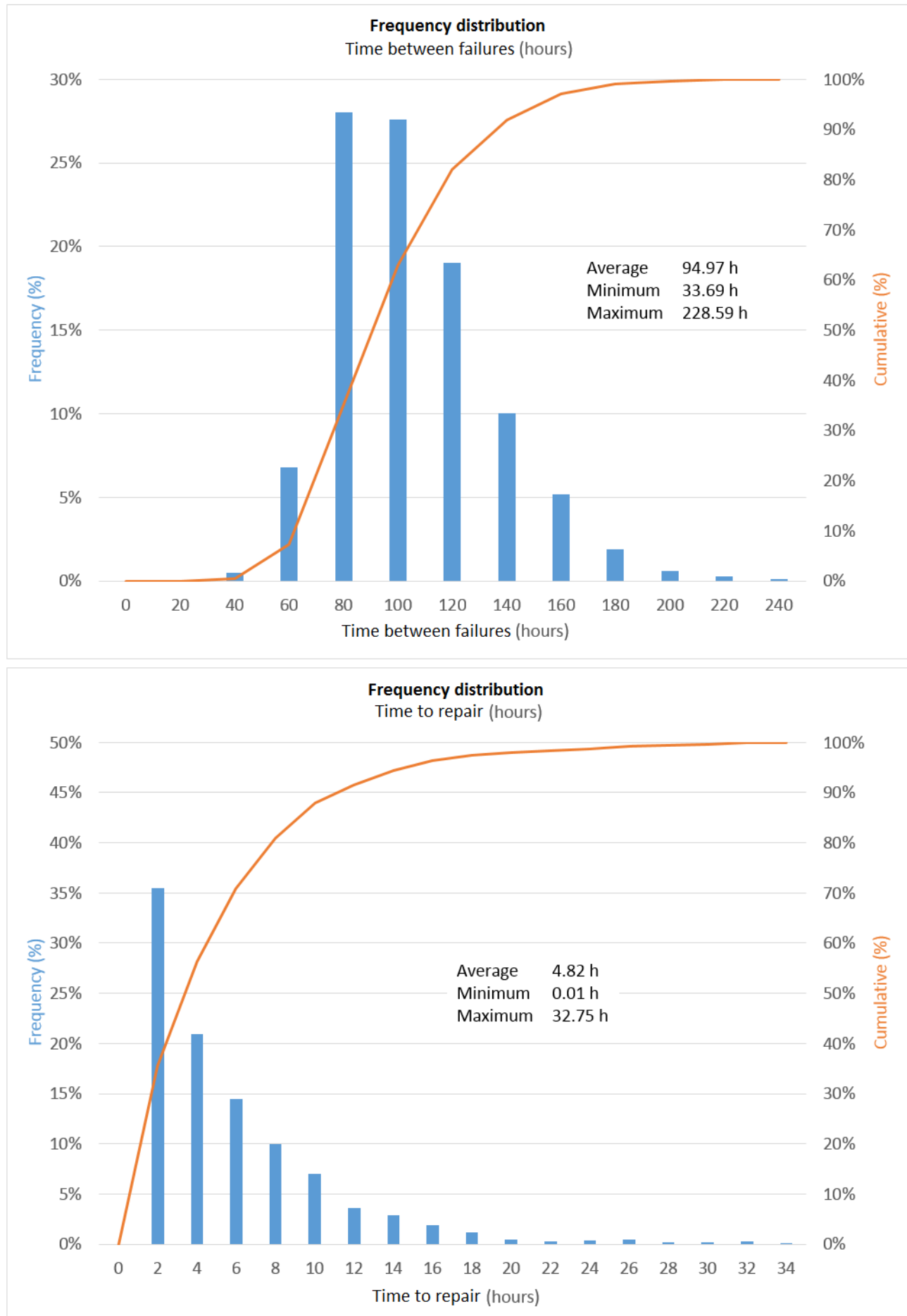

Figure 1 Simulation model output for the crusher with $95 \%$ availability 
The simulation output shows that the average values for TBF and TTR are close to the expected values of 100 hours and 5 hours, respectively, but variation in the observed values is quite significant - with TBF ranging between 33 and 299 hours and TTR between 0 and 33 hours. This highlights the difference between using average values and using data that includes variability.

Revisiting the original example where we had a 2,000 t/h crusher with $95 \%$ availability, what is the actual production rate achieved by the crusher? This is shown in Figure 2.

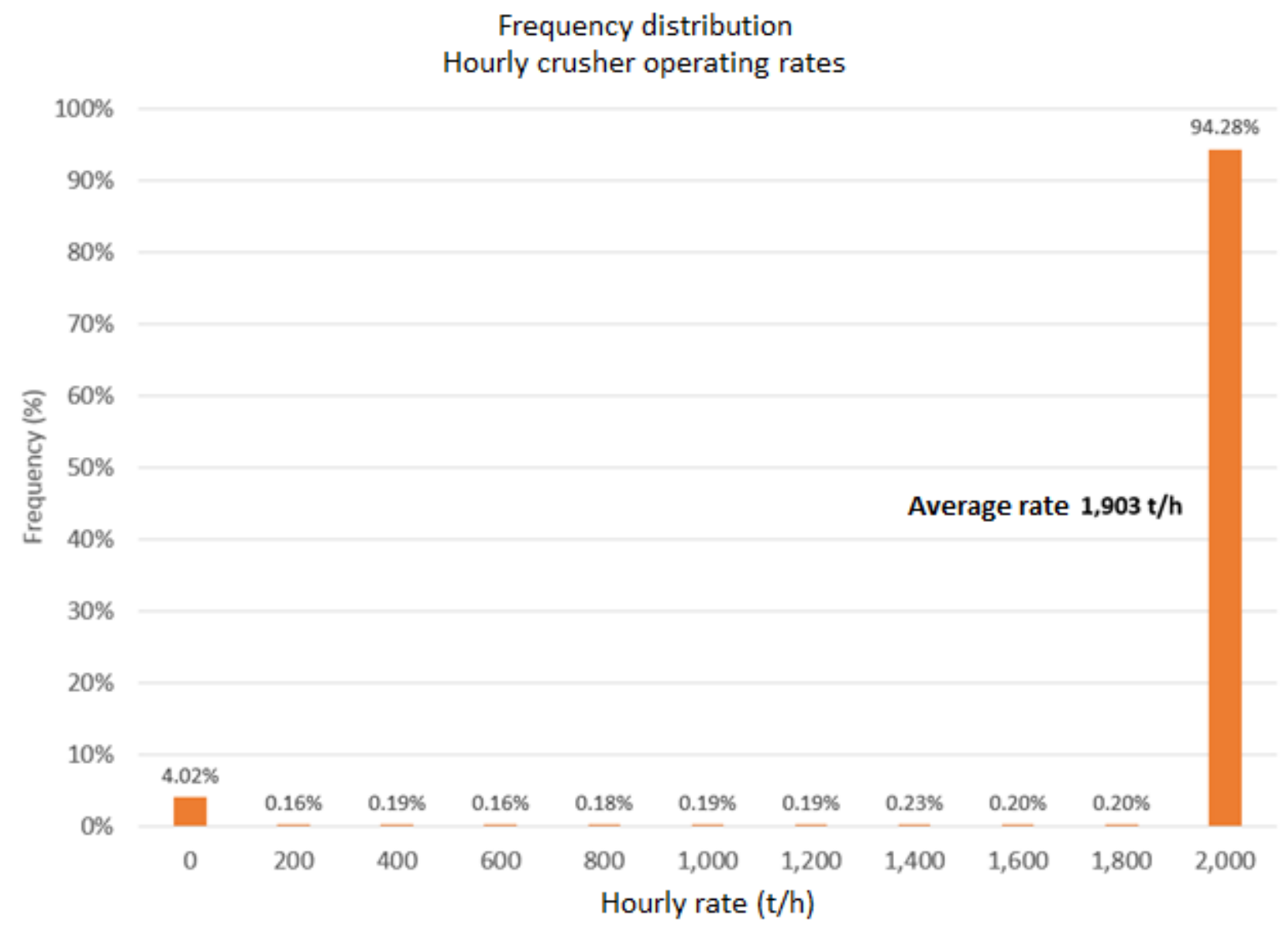

Figure 2 Simulation model output for the crusher with operating rates

The dynamic model of the crusher shows that the average rate achieved was $1,903 \mathrm{t} / \mathrm{h}$, which is the same as that predicted by the static calculation. However, the dynamic model also reveals the frequency distribution of the partial rates achieved by the crusher on an hourly basis resulting from the occurrences of failures and the times to repair. These results are from a very simple model of one piece of equipment running at $100 \%$ when available and at $0 \%$ during failures, i.e. either on or off, which is why the model results match those of the static calculation. 


\section{$4 \quad$ Expanding the model and adding complexity}

The simple model example, described in Section 3, included one piece of equipment - the crusher - and thus it did not take into account any effects from upstream or downstream operations or its interaction with other operations within the mine. If the model is expanded to include a larger portion of the process, e.g. mine production and haul trucks dumping into the crusher and ore handling conveyors, and the process plant taking ore away, the results for crusher performance and utilisation will include the effects of:

- Stope production.

- Haul truck transportation to the crusher (including effects of the haul truck fleet size, cycle times, queuing, payload etc.).

- Operating schedules for crews (including shift changes, breaks, safety meetings etc.).

- Ore handling rates of conveyors and other equipment (installed capacities for conveyors and storage bins etc.).

- Availability of conveyors and other equipment (maintenance schedules and failure distributions).

A simplified flow diagram of the expanded model is shown in Figure 3.

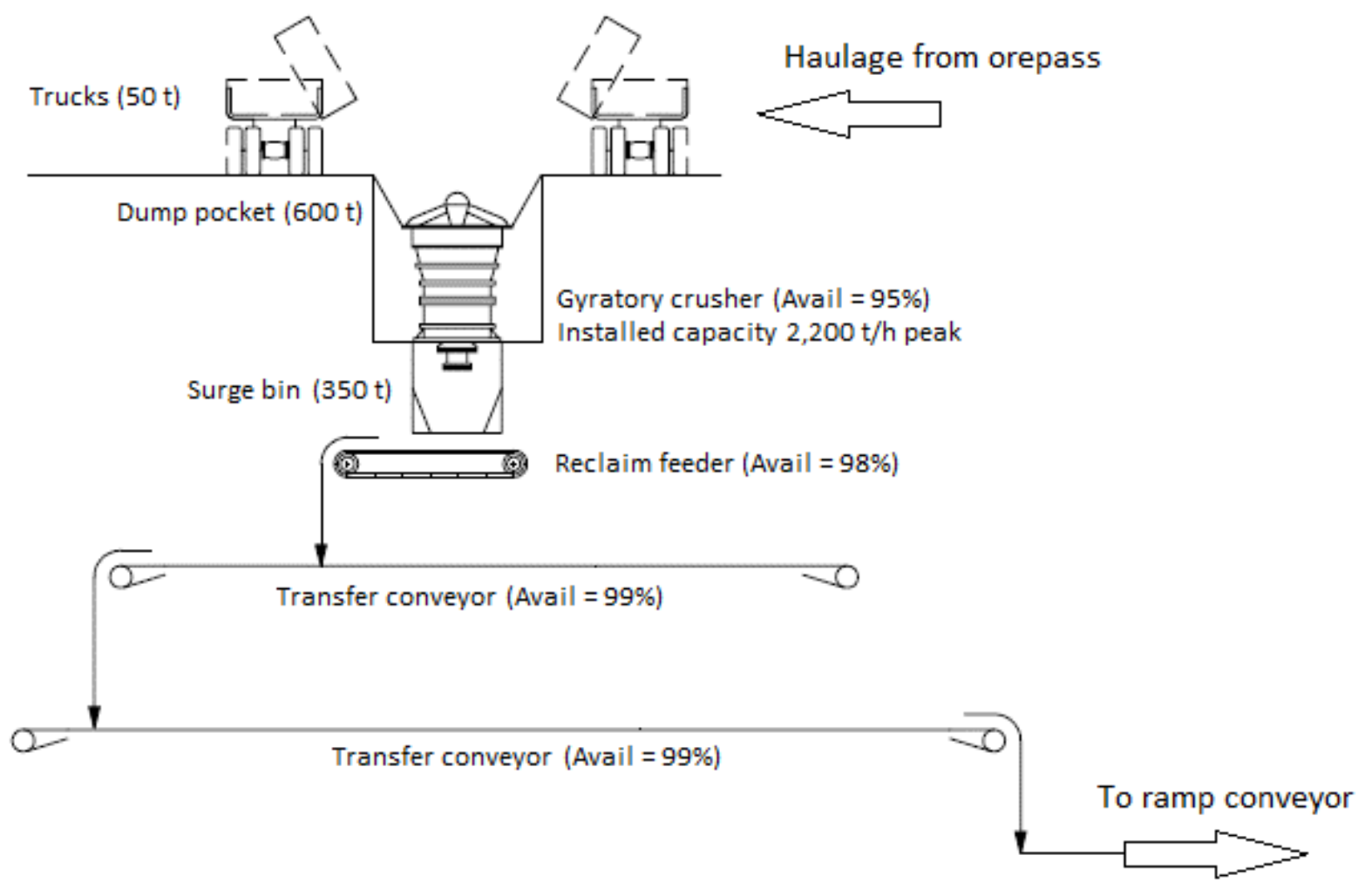

Figure 3 Flow diagram for underground crusher including upstream and downstream operations

With the model configured as such, the performance of the crusher will depend on ore production in the mine, the presence of muck in the orepasses, the efficiency of the haul trucks feeding the crusher, the availability of the crusher and downstream equipment, the operating schedule of the mine etc.

The model output for hourly crusher rate with these additional factors is shown in Figure 4. 


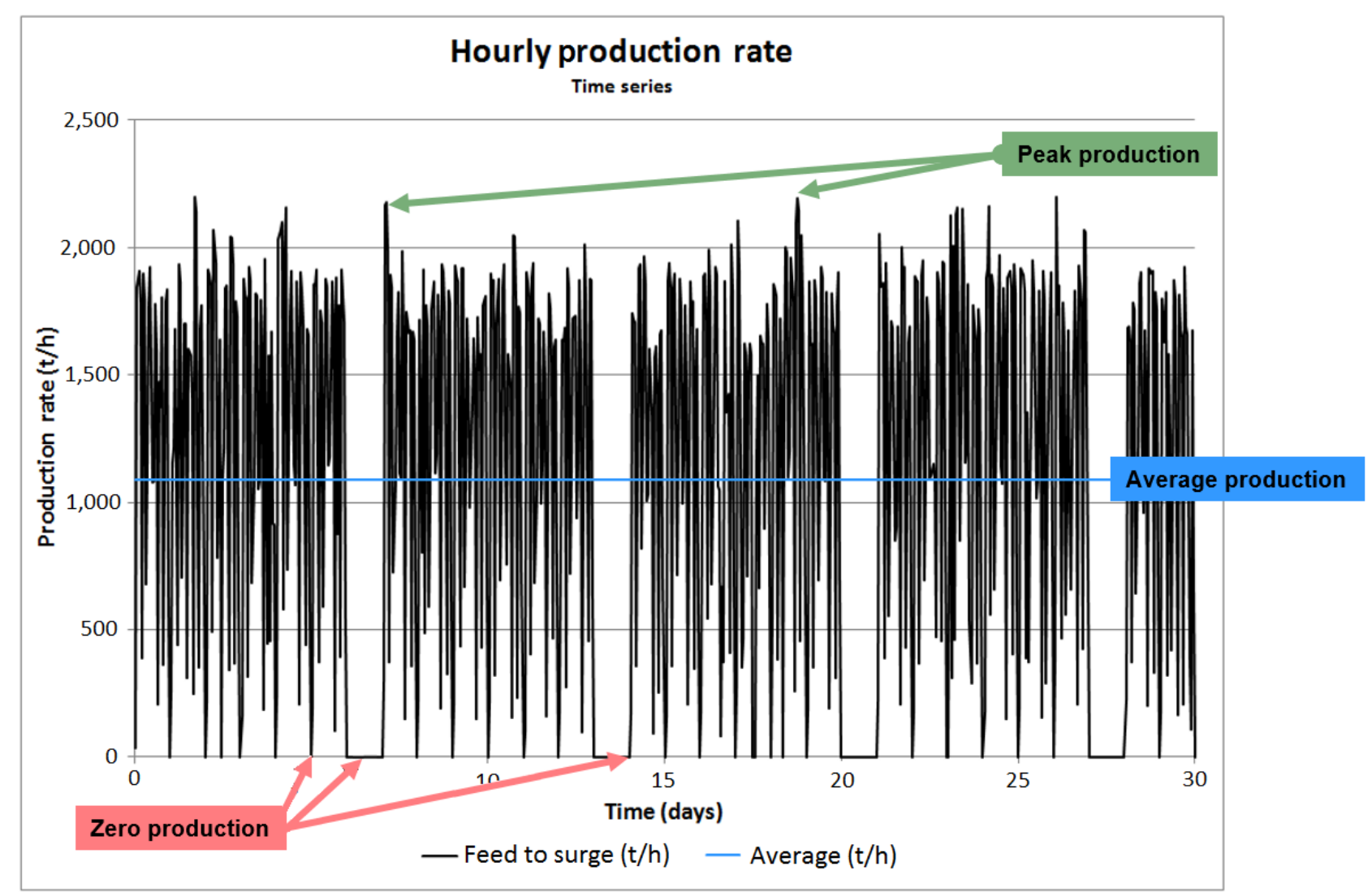

Figure 4 Hourly crusher rates over a 30-day period (with upstream and downstream effects)

The above graph illustrates the wide variation in crusher production rates over a 30-day period. Note that, in this particular case, the mine operated on a 6-days per week schedule, which accounts for the zero rates every seventh day. The main point to be made here is that, even though the installed crusher rate is $2,200 \mathrm{t} / \mathrm{h}$, the average rate over the period was about $1,100 \mathrm{t} / \mathrm{h}$, indicating a utilisation of about $50 \%$.

The same data is presented as a frequency distribution in Figure 5. 


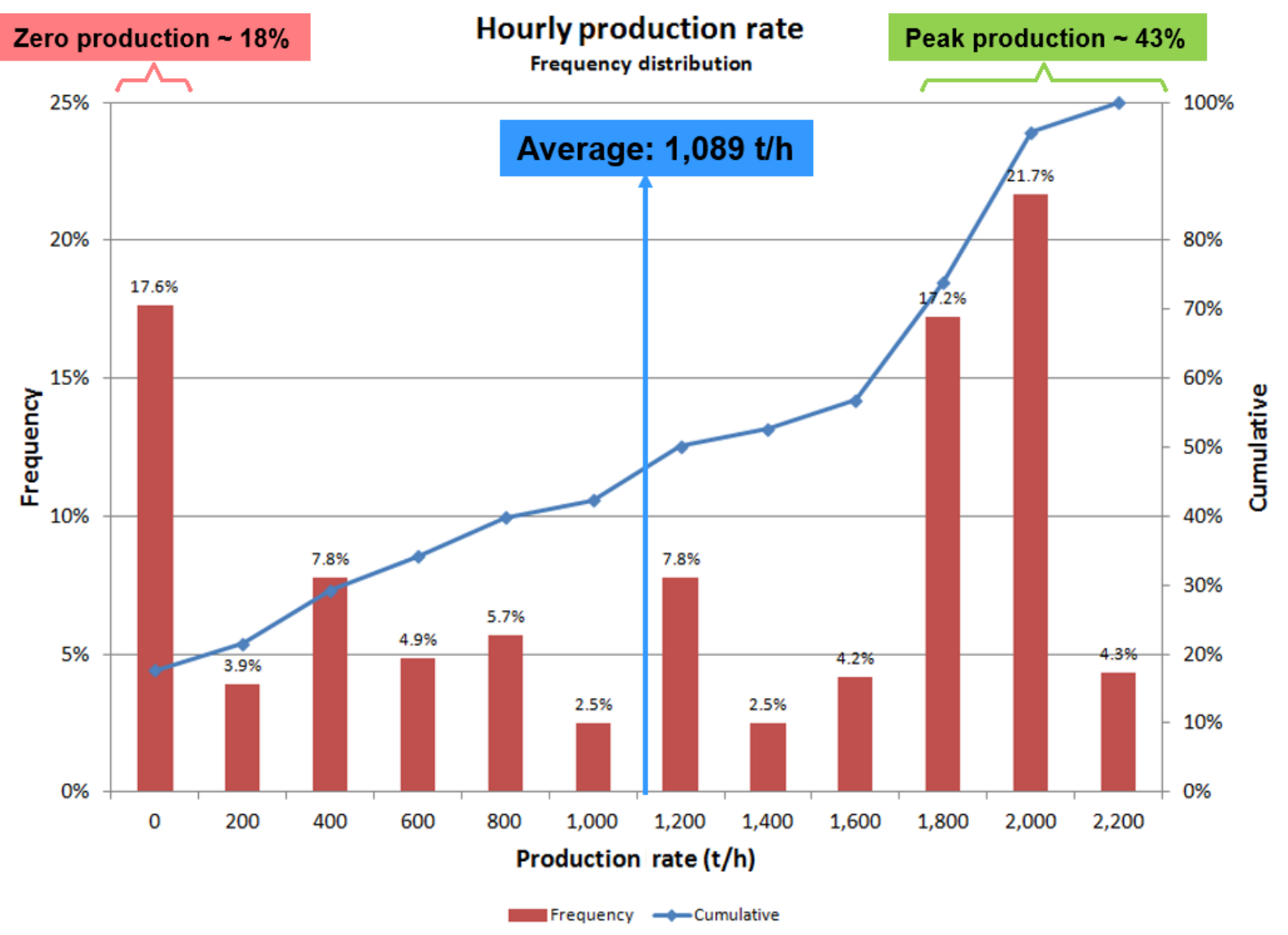

Figure 5 Distribution of hourly crusher rates over a 30-day period

The histogram above illustrates a few interesting points:

- Peak production (assuming rates of $1,800 \mathrm{t} / \mathrm{h}$ or higher) occurred $43 \%$ of the time.

- Zero production occurred $18 \%$ of the time; given that the mine produces six days per week and one day in seven accounts for $14 \%$, this leaves $4 \%$ when the mine was operating but the crusher was not.

- The remaining $39 \%$ of the time, the crusher was operating at partial rates. This could have been due primarily to interaction between system components beyond the crusher itself. For example, the mine not providing enough ore, insufficient trucks hauling from the orepasses, maintenance or breakdowns on conveyors or other equipment, shift changes, meal breaks or other manning interruptions.

Given the above data, you could come to one or more of the following conclusions:

- This $2,200 \mathrm{t} / \mathrm{h}$ crusher is only going to operate at an average rate of $1,100 \mathrm{t} / \mathrm{h}$.

- This crusher is only going to operate at its installed rate $43 \%$ of the time.

- This crusher is going to be operating at reduced rates $39 \%$ of the time, and $18 \%$ of the time it will not be operating at all.

- The process plant only needs to be designed to handle $1,100 \mathrm{t} / \mathrm{h}$ on average. Or, alternatively, the downstream process plant, designed for $1,500 \mathrm{t} / \mathrm{h}$, will not receive the required amount of ore. 
At this point, if these results are not optimal, the model could be used to test methods to improve the performance of the system. For example, the inputs to the model could be changed to test the effect of the following:

- Operate the mine seven days per week instead of six.

- Reduce the time lost to shift changes and breaks by staggering crews.

- Change the truck parameters, e.g. increase the size of the truck fleet, change the payload of the trucks etc.

- Change the availability assumptions for the ore handling equipment, i.e. change the maintenance strategy or install more reliable equipment etc.

- Add spare equipment.

Typically, each of the changes to the model input above would be done individually to quantify the incremental change resulting from each modification. Once this data is available, the benefits (or consequences) can be ranked to create a list of potential design changes that would provide the highest returns costs and on investment. By doing this, the model results can be used to evaluate design and capital investment decisions, and create a 'road map' towards an optimal design.

\section{$5 \quad$ Applications to underground mine design}

\subsection{Room and pillar mining}

A 3,000 t/d underground room-and-pillar diamond mine was planned in Northern Canada (Quan \& Durston 2003). The mine was to be a complex network of underground mining activities, with separate crews performing drilling, blasting, loading and haulage, ground support, and backfill operations. Ore was to be hauled to the surface by truck and/or underground conveyor, where it would be stored in surface stockpiles or bins. The diamonds were to be extracted from the ore in a process plant and the processed waste material was to be converted to paste and pumped to a surface disposal site or back to the underground workings for use as backfill.

To determine equipment and labour requirements to meet the mine production rate, a static analysis (spreadsheet-type) was originally conducted using average values for haul distances, productive hours per shift, equipment availability, etc. While this was adequate for an initial evaluation of the design, the static analysis did not provide any insight into the peaks and valleys of mine production and ore processing.

Without this insight, many operational questions could not be answered:

- What would be the expected daily maximums and minimums?

- How many crews would be needed to maintain a minimum production rate in all cases?

- How much storage capacity would be required on the surface to buffer any surges/interruptions from the mine?

- Would there be any issues with congestion at the ore dump points?

- What would be the consequences of equipment failures?

To answer these, and many other questions, a dynamic simulation model was developed to represent the major components of the system and their interaction. The model included all underground unit operations and the surface paste plant. The model was designed to assist in:

- Determining the equipment production rates and the number of equipment units required.

- Assessing the sensitivity of production rates to changes in major operating parameters. 
- Identifying system bottlenecks, which for those unfamiliar with the Theory of Constraints are the critical points in the system where improvements (process rate, storage capacity etc.) act to improve the overall system.

- Evaluating the interaction between the underground operations and the paste plant.

- Confirming operational philosophies, such as the number of working hours per shift, scheduling of planned maintenance etc.

The modelling was performed during the early feasibility study phase of the project and included these major unit operations and area activities:

- Underground mining operations:

$\circ$ Drilling.

○ Charging of explosives.

○ Blasting.

$\bigcirc$ Mucking.

- Installation of ground support.

○ Placement of fill.

- Underground storage in orepasses and coarse ore bins above the primary crushers.

- Surface ore storage.

- General process plant operations, focusing on the production of paste fill. Other unit operations within the process plant were not included in the simulation study.

- Materials handling:

○ Trucking ore.

- Conveying ore and waste rock.

- Distributing paste fill through pipelines.

The simulation study was performed for a target production rate of $3,000 \mathrm{t} / \mathrm{d}$ of ore delivered to the surface process plant. Overall system performance was affected by statistical variations in unit operation, mechanical availability, throughput, and operating and system management strategies, such as conveyor control, dispatch logic, and bin and stockpile management.

The model output for daily mine production is shown in Figures 6 and 7.

The model results below are from a model input configuration that achieved the target production rate of $3,012 \mathrm{t} / \mathrm{d}$ with daily variations ranging from 2,200 to $4,400 \mathrm{t} / \mathrm{d}$. These variations were the result of inputs for mobile equipment fleets, drawpoint locations relative to the ore delivery points, equipment availability, operating schedules, paste production etc. 


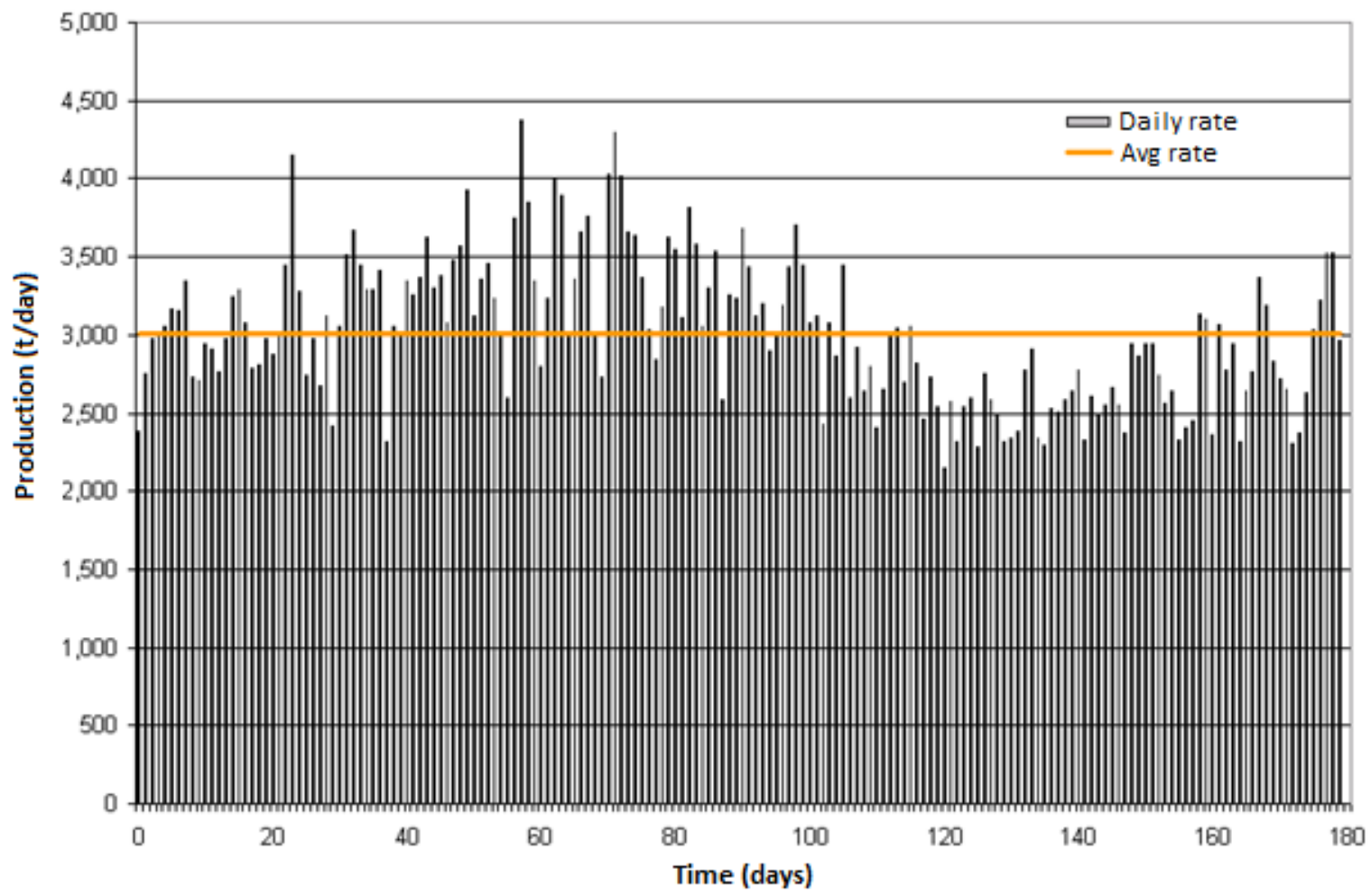

Figure 6 Daily mine production over 180 days

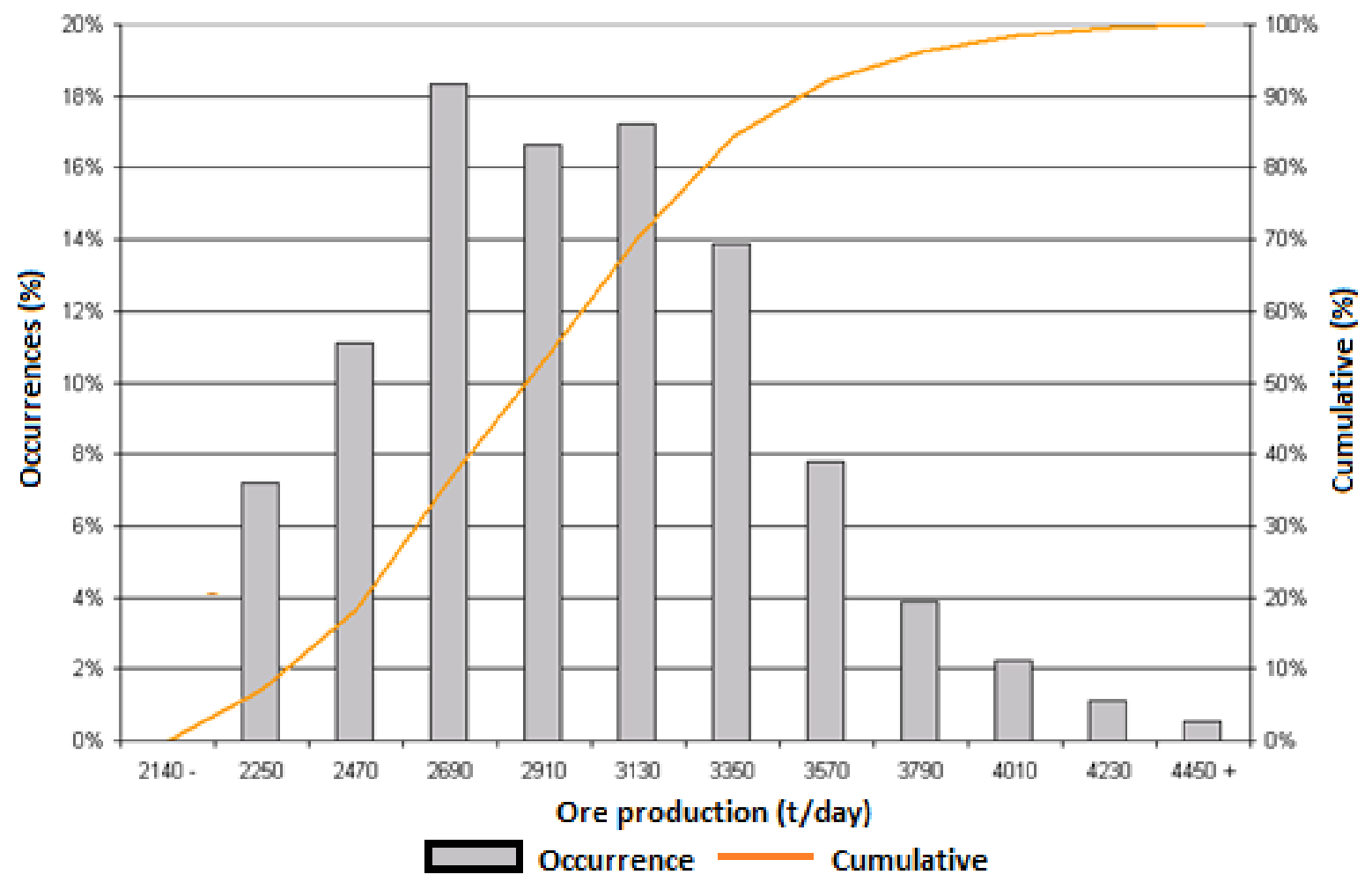

Figure 7 Distribution of daily ore production rate over 180 days

The net results of the analysis are the following conclusions:

- Verification of the mining method.

- Number of active mining panels required. 
- Underground coarse ore bin capacity.

- Orepass capacities of 100 tonnes.

- Surface storage capacity.

- Number of single-boom electric-hydraulic drill jumbos required per panel.

- Number of $2.8 \mathrm{~m}^{3}$ diesel LHDs required for each panel.

- Number of diesel haulage trucks required as the locations of the active mining panels relative to the orepasses change with time.

These determinations were based on unit productivities appropriate for the selected mining method and the indicated mining conditions, and on performance and availabilities typical for the major mining equipment selected. They are also cognisant of the interdependency of the various mining activities. The simulation model was able to provide insight into the complex behaviour of the mining, processing, and backfilling operations of the project - insight that was not available from a static spreadsheet analysis. While the average values for equipment fleet sizes were similar in the static and dynamic model outputs, the dynamic model provided additional information on what the peak equipment requirements would be and how often.

\subsection{Underground haulage and traffic congestion}

Underground haulage is usually a complex network of mobile equipment dedicated to mine development, production and support services. Typically, broken rock is produced using mobile equipment (drills and explosive charge-up trucks or continuous miners) at the working face and moved by LHDs to haul trucks (or to conveyors) either directly or via orepasses depending on the geometry of the mine. The distances travelled by mobile equipment, the size of each fleet, the layout of the haulage network, and the rules for traffic control, all contribute to the overall productivity of the mine as a system.

A discrete-event simulation model of an underground ramp haulage network at an underground zinc mine was created to estimate the potential effects of traffic congestion on mine production for various haulage scenarios. Haulage of ore would be from multiple levels via a single access ramp to a crusher or to intermediate dumping bays. The crushed ore would be conveyed to one of several underground silos and then hoisted to the surface where it is stockpiled.

The model included underground truck haulage of run-of-mine material from several production levels to the crusher plus additional mobile equipment such as drill jumbos, working platforms, and light vehicles with haulage speeds assigned to each vehicle type. Various options for vehicle passing bay spacing and rules for truck priority were examined.

The model was used to test several scenarios, including combinations of:

- Different throughput targets.

- Truck payload and type (diesel versus electric).

- Spacing for vehicle pull-out areas (passing bays).

- Rules for vehicle priority.

- Effects of other mobile equipment fleets.

- Use of traffic control lighting to change truck priority rules.

- Allowance for 'tailgating' by haul trucks.

For each case, the model provided the potential production capacity, including impacts from traffic congestion, such that the optimum truck fleet requirements and operating strategy could be identified for each production scenario. 
For example, in Figure 8 below, the results for mine annual throughput as a function of truck fleet size and truck payload size are shown.

\section{Truck payload analysis}

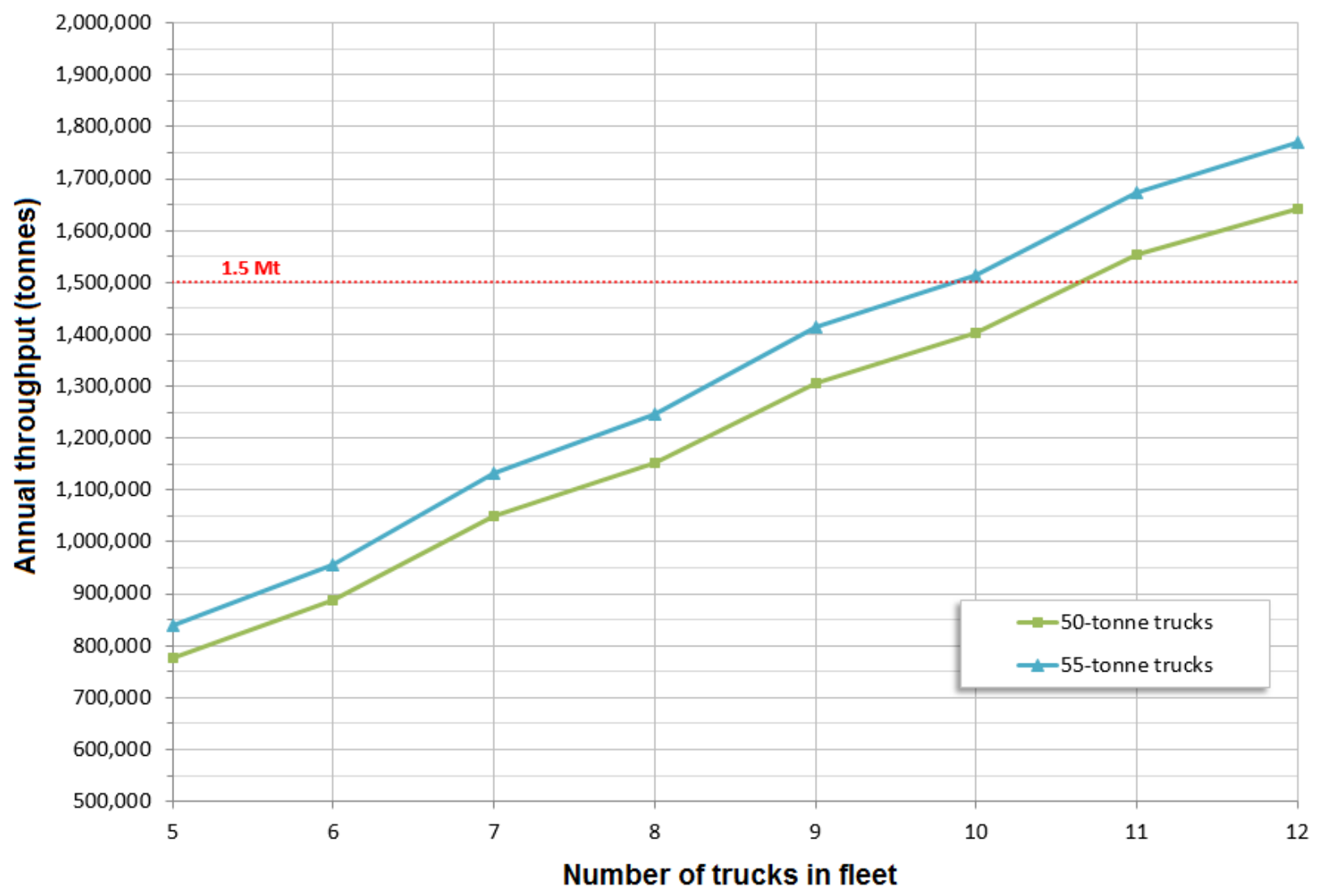

Figure 8 Model results for throughput versus truck fleet size and truck payload size

The above results show the effect on the system of changing only one variable - the truck payload. The incremental increase in production rate that can be achieved using the 55 tonne truck (rather than the 50 tonne truck) enables the target production rate of $1.5 \mathrm{Mt} / \mathrm{y}$, to be reached utilising one less truck.

To adequately simulate haulage, the model included the truck haulage ramp geometry - segment length, ramp gradient, locations of passing bays and entrances to the production levels - rules for priority on the ramp (rights-of-way), and various types of mobile equipment. Depending on the model inputs for traffic rules, vehicles encountering other vehicles while in transit would either yield or take the right-of-way at passing bays and production level crosscuts. The spacing of passing bays was a user-definable option. In general, loaded haul trucks had the right-of-way in all cases, but some exceptions to this rule were tested during analysis.

Figure 9 shows the model outputs for mine production as a function of truck fleet size and passing bay spacing. 


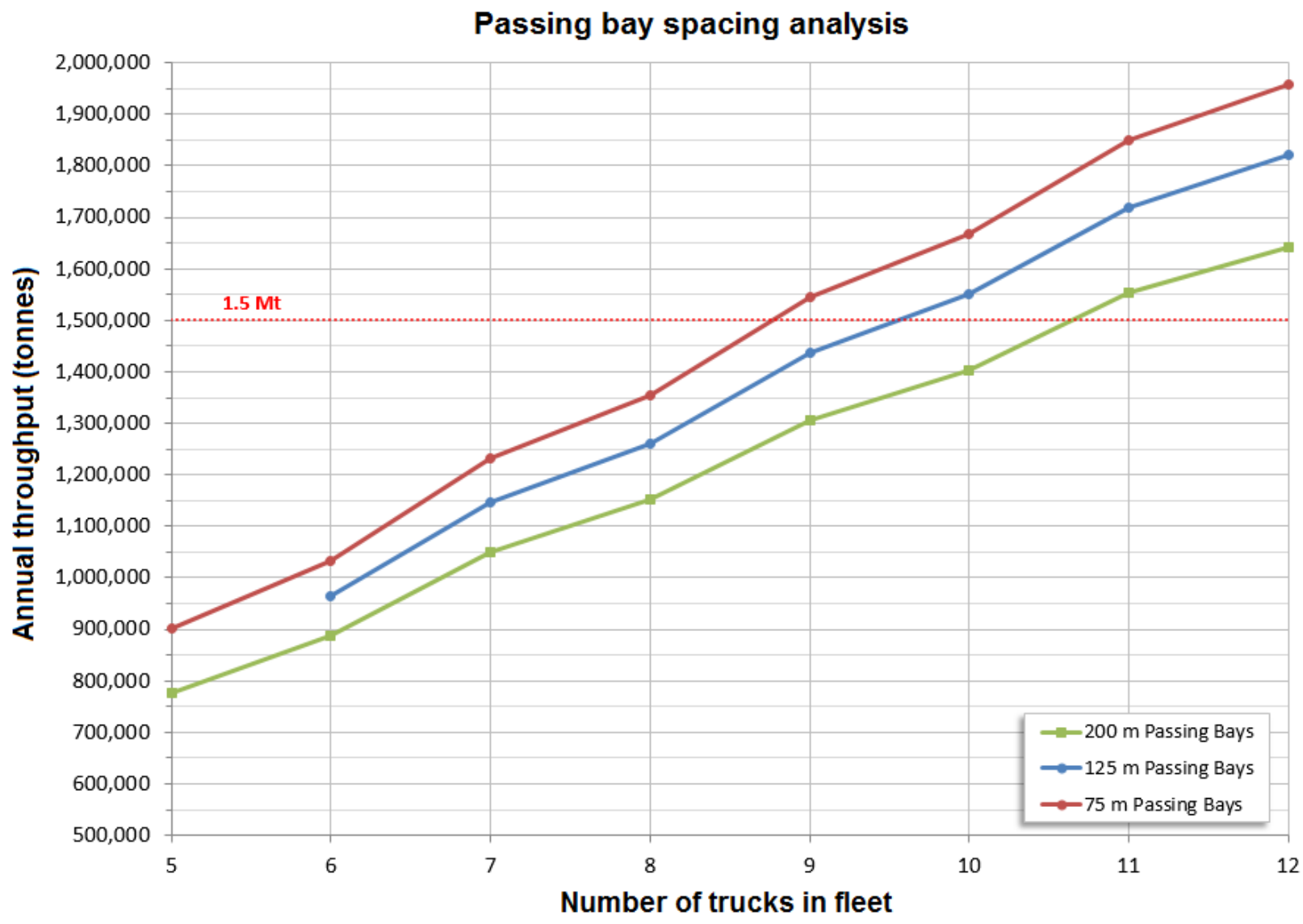

Figure 9 Model results for throughput versus truck fleet size and passing bay spacing

Although the results are intuitive (more frequent passing bays results in less traffic congestion), the model results quantify this benefit, allowing a cost/benefit analysis to be conducted and minimising capital expenditure.

A final example demonstrates the ability of the model to test the benefits of a change in operating strategy, which typically does not require a significant change in capital cost. Figure 10 shows the model results for a series of runs that tested the effects of productive time per shift. 


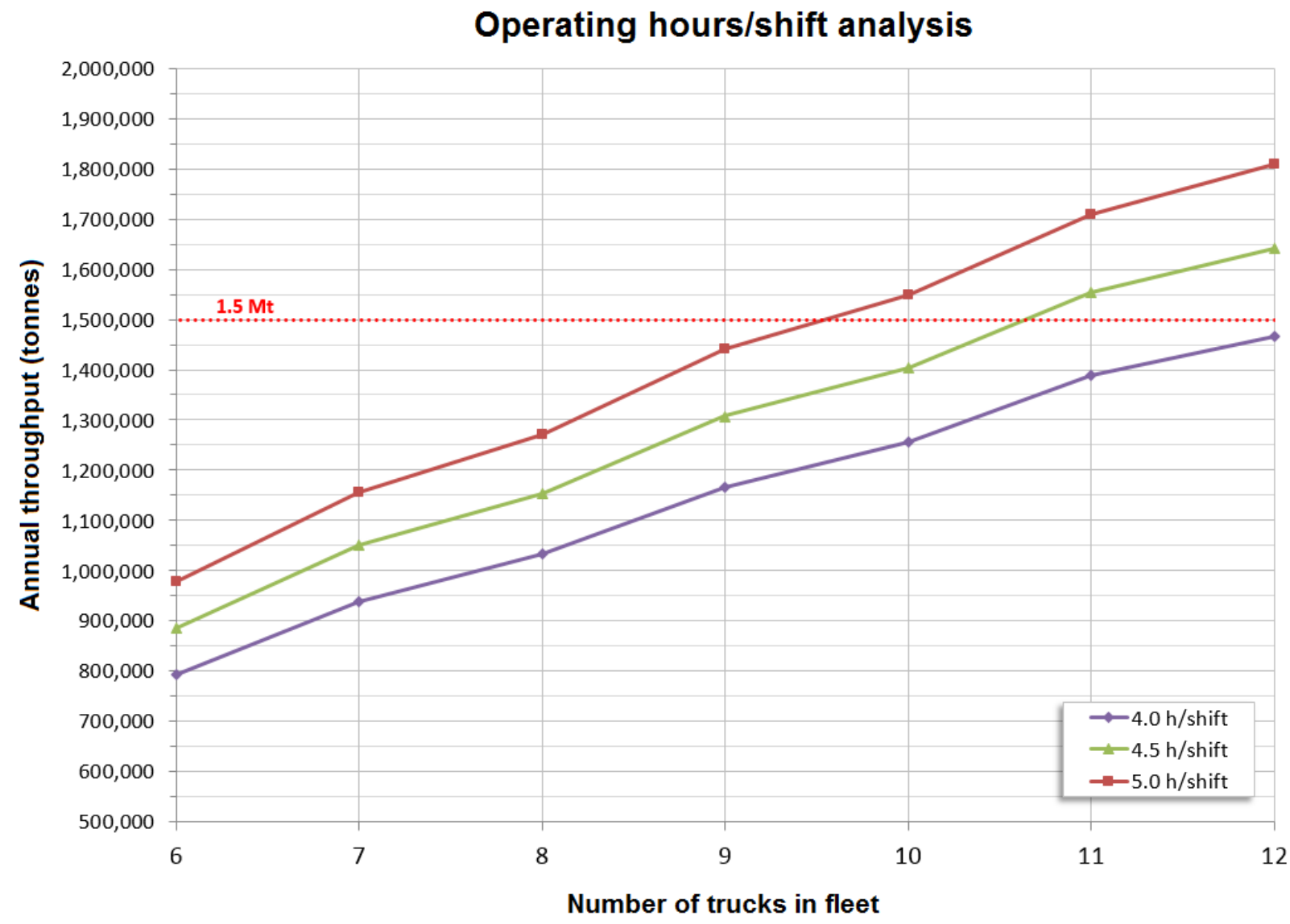

Figure 10 Model results for throughput versus truck fleet size and productive hours per shift

The results above identify the potential increases, or decreases, in mine productivity stemming from a half hour increase, or decrease, in productive time per shift ( $4.5 \mathrm{~h}$ per $8 \mathrm{~h}$ shift being the base case assumption). This illustrates the usefulness of a model in testing changes to operating philosophy rather than only making changes to the design of capital infrastructure.

\subsection{Ore handling and processing}

Run-of-mine ore handling, whether by mobile equipment or by continuous means, typically requires large amounts of capital equipment. In continuous systems, bins, feeders and conveyors are installed underground and can transport ore thousands of metres to the surface via declines or to skip hoists in production shafts. Once on the surface, the ore continues into the process plant where it is converted into products for market.

One question that is always asked is 'Will this design be able to meet the target production rates?'

Models are typically built from mass balances, process flow diagrams, installed equipment capacities, and operational details. Any supporting processes and resources, like utilities and maintenance crews are also included. The model becomes a process simulator that mimics the operation of the system over time and allows us to:

- Validate system behaviour to be as intended and evaluate performance.

- Optimise design parameters (storage size, process rates) via sensitivity analysis to maximise marginal benefit (most efficient allocation of capital).

- Streamline operations, identify bottlenecks and reduce excess capacity to increase system efficiency. 
- Rank equipment by criticality to the system to identify top downtime contributors and devise risk management techniques.

- Identify planned and unplanned maintenance demands and evaluate whether there is sufficient equipment sparing and redundancy.

- Align maintenance activities across different areas of the operation, e.g. the mine and the plant.

- Test alternate system configurations and operations management techniques to determine the most cost effective design to meet production requirements.

- Quantify risk to justify design decisions and capital investment.

These types of models, often called RAM models because they assess reliability, availability and maintainability of a process (Sage \& Rouse 2014), can provide very rich detail about how a process will operate over time. In addition to the kinds of data that have been shown already, the model output can quickly identify problem areas in the design.

For example, in Figure 11 below, the performance of a portion of an underground ore handling system is tabulated by equipment.

\begin{tabular}{|c|c|c|c|c|c|c|}
\hline ID no. & Equipment name & Running & Blocked & Starved & Failure & Mtc \\
\hline \multicolumn{7}{|l|}{ Crusher 1} \\
\hline 101 & Gyratory crusher no. 1 & $57.4 \%$ & $6.9 \%$ & $29.5 \%$ & $0.2 \%$ & $6.0 \%$ \\
\hline 102 & Crusher surge bin feeder no. 1 & $57.4 \%$ & $9.6 \%$ & $26.5 \%$ & $0.5 \%$ & $6.0 \%$ \\
\hline 104 & Transfer conveyor no. 1 & $65.6 \%$ & $5.7 \%$ & $21.8 \%$ & $0.9 \%$ & $6.0 \%$ \\
\hline \multicolumn{7}{|l|}{ Crusher 2} \\
\hline 201 & Gyratory crusher no. 2 & $56.8 \%$ & $7.7 \%$ & $29.1 \%$ & $0.2 \%$ & $6.2 \%$ \\
\hline 202 & Crusher surge bin feeder no. 2 & $56.8 \%$ & $11.9 \%$ & $24.7 \%$ & $0.3 \%$ & $6.2 \%$ \\
\hline \multicolumn{7}{|c|}{ Inclined belts } \\
\hline 106 & Incline conveyor no. 1 & $70.3 \%$ & $5.1 \%$ & $19.7 \%$ & $1.1 \%$ & $3.8 \%$ \\
\hline 208 & Incline conveyor no. 2 & $50.0 \%$ & $2.6 \%$ & $42.5 \%$ & $1.1 \%$ & $3.8 \%$ \\
\hline \multicolumn{7}{|c|}{ Skips 1 and 2} \\
\hline 113 & Shaft 2 ore reclaim feeder no. 1 & $80.0 \%$ & $4.4 \%$ & $4.4 \%$ & $0.4 \%$ & $10.9 \%$ \\
\hline 213 & Shaft 2 ore reclaim feeder no. 2 & $1.9 \%$ & $76.7 \%$ & $10.0 \%$ & $0.5 \%$ & $10.9 \%$ \\
\hline 114 & Shaft 2 loadout conveyor & $81.9 \%$ & $4.1 \%$ & $2.7 \%$ & $0.4 \%$ & $10.8 \%$ \\
\hline 121 & Skip no. 1 and 2 & $81.9 \%$ & $2.9 \%$ & $3.2 \%$ & $1.1 \%$ & $10.8 \%$ \\
\hline \multicolumn{7}{|c|}{ Skips 3 and 4} \\
\hline 313 & Shaft 3 ore reclaim feeder no. 1 & $42.3 \%$ & $4.0 \%$ & $42.1 \%$ & $0.5 \%$ & $11.1 \%$ \\
\hline 316 & Shaft 3 ore reclaim feeder no. 2 & $56.8 \%$ & $4.0 \%$ & $28.2 \%$ & $0.4 \%$ & $10.6 \%$ \\
\hline 314 & Shaft 3 loadout conveyor no. 1 & $73.7 \%$ & $3.8 \%$ & $10.3 \%$ & $1.1 \%$ & $11.1 \%$ \\
\hline 221 & Skip no. 3 and 4 & $73.7 \%$ & $2.8 \%$ & $11.4 \%$ & $1.0 \%$ & $11.1 \%$ \\
\hline \multicolumn{7}{|c|}{ Skips 5 and 6} \\
\hline 513 & Shaft 3 ore reclaim feeder no. 3 & $31.4 \%$ & $42.4 \%$ & $14.6 \%$ & $0.5 \%$ & $11.1 \%$ \\
\hline 516 & Shaft 3 ore reclaim feeder no. 4 & $21.4 \%$ & $55.0 \%$ & $12.6 \%$ & $0.4 \%$ & $10.6 \%$ \\
\hline 514 & Shaft 3 loadout conveyor no. 2 & $78.2 \%$ & $3.7 \%$ & $6.7 \%$ & $0.9 \%$ & $10.5 \%$ \\
\hline 321 & Skip no. 5 and 6 & $78.2 \%$ & $2.7 \%$ & $7.7 \%$ & $0.8 \%$ & $10.5 \%$ \\
\hline \multicolumn{7}{|c|}{ Surface belts } \\
\hline 152 & Shaft 2 discharge conveyor & $52.9 \%$ & $1.7 \%$ & $33.5 \%$ & $1.1 \%$ & $10.8 \%$ \\
\hline 252 & Shaft 3 discharge conveyor no. 1 & $63.0 \%$ & $1.8 \%$ & $23.1 \%$ & $1.0 \%$ & $11.1 \%$ \\
\hline 352 & Shaft 3 discharge conveyor no. 2 & $66.8 \%$ & $1.7 \%$ & $20.1 \%$ & $0.9 \%$ & $10.5 \%$ \\
\hline 153 & Coarse ore overland conveyor & $60.9 \%$ & $1.0 \%$ & $26.7 \%$ & $0.9 \%$ & $10.5 \%$ \\
\hline 154 & Surface stacker & $60.9 \%$ & & $27.6 \%$ & $1.0 \%$ & $10.5 \%$ \\
\hline
\end{tabular}

Figure 11 Model results for equipment utilisation in an underground ore handling system

The model clearly shows that the highest utilisation in the system is around the three hoists, which are each running at about $80 \%$ utilisation. The model output also shows the portion of time that the hoist is 
starved, indicating upstream interruptions, and blocked, indicating downstream interruptions. Other time usage statistics such as downtimes for failures and maintenance are also shown.

At first view, these results indicate that the mine is delivering ore to the gyratory crushers (ID 101 and 201) at a rate that underutilises the crusher. However, further inspection reveals that the utilisation of the crushers, which are operating at about $58 \%$ utilisation, is consistent with the operating assumption that trucks are delivering ore to the crushers approximately 8 hours in each 12 hour shift and are not present at the crusher $100 \%$ of the time, i.e. there are times when there are no trucks dumping.

In this particular case, the results identify the potential for increased throughput by increasing the amount of ore delivered to the hoists to reduce the hoist starving times. At this point, the model inputs can be modified to increase the number of productive trucking hours in a shift, increase the size of the storage bins between the crushers and the hoists, modify rate inputs, change availability assumptions etc. to 'unlock' some unrealised capacity.

Applying criticality ranking to the model output is another useful feature of simulation modelling shown in Figure 12 with the example of data from a model of a potash processing plant.

\begin{tabular}{|c|c|c|c|}
\hline & & Contaifuring & \\
\hline & & $-\mathrm{NaCl}$ Centrifuge Train A & $\begin{array}{l}4.0 \% \\
2.0 \%\end{array}$ \\
\hline & & Clarifier U/F Pump & $0.2 \%$ \\
\hline & & $\mathrm{KCl}$ Crystalliser Pump & $0.2 \%$ \\
\hline & & $\mathrm{KCl}$ Crystalliser Pump Box & $0.0 \%$ \\
\hline & & $\mathrm{NaCl}$ Centrifuge Cake Drag Conveyor & $0.1 \%$ \\
\hline Plant Area & Impact & $\mathrm{NaCl}$ Centrifuge Centrate Pump & $0.2 \%$ \\
\hline Centrifuge \& Drying & $3.1 \%$ & $\mathrm{NaCl}$ Centrifuge Centrate Pumpbox & $0.0 \%$ \\
\hline Crystallisation & $0.9 \%$ & $\mathrm{NaCl}$ Centrifuge Feed Tank & $0.0 \%$ \\
\hline Evaporation & $4.1 \%$ & $\mathrm{NaCl}$ Centrifuge Holding Tank & $0.0 \%$ \\
\hline Granular Production Line & $0.0 \%$ & $\mathrm{NaCl}$ Centrifuge Holding Tank Agitator & $0.0 \%$ \\
\hline Other & $0.3 \%$ & $\mathrm{NaCl}$ Centrifuge Tank Pump & $0.3 \%$ \\
\hline $\mathrm{NaCl}$ Centrifuging & $4.0 \%$ & $\mathrm{NaCl}$ Centrifuge 1 & $0.2 \%$ \\
\hline Primary Mining & $0.6 \%$ & Hydrocyclone & $0.0 \%$ \\
\hline Product Screening & $1.3 \%$ & $\mathrm{NaCl}$ Centrifuge 2 & $0.5 \%$ \\
\hline Secondary Mining & $1.0 \%$ & Clarifier Feed Launder & $0.0 \%$ \\
\hline Standard Production Line & $1.5 \%$ & Clarifier Rake & $0.1 \%$ \\
\hline Utilities & $0.4 \%$ & $\ominus \mathrm{NaCl}$ Centrifuge Train B & $2.0 \%$ \\
\hline Waste Salt Management & $0.1 \%$ & Clarifier U/F Pump & $0.2 \%$ \\
\hline Model Block & $0.0 \%$ & $\mathrm{KCl}$ Crystalliser Pump & $0.2 \%$ \\
\hline Saturation & $0.0 \%$ & $\mathrm{KCl}$ Crystalliser Pump Box & $0.0 \%$ \\
\hline Grand Total & $17.2 \%$ & $\mathrm{NaCl}$ Centrifuge Cake Drag Conveyor & $0.2 \%$ \\
\hline & & $\mathrm{NaCl}$ Centrifuge Centrate Pump & $0.2 \%$ \\
\hline & & $\mathrm{NaCl}$ Centrifuge Centrate Pumpoox & $0.0 \%$ \\
\hline & & $\mathrm{NaCl}$ Centrifuge Feed Tank & $0.0 \%$ \\
\hline & & $\mathrm{NaCl}$ Centrifuge Holding Tank & $0.0 \%$ \\
\hline & & $\mathrm{NaCl}$ Centrifuge Holding Tank Agitator & $0.0 \%$ \\
\hline & & $\mathrm{NaCl}$ Centrifuge Tank Pump & $0.3 \%$ \\
\hline & & $\mathrm{NaCl}$ Centrifuge 1 & $0.2 \%$ \\
\hline & & Hydrocyclone & $0.0 \%$ \\
\hline & & $\mathrm{NaCl}$ Centrifuge 2 & $0.5 \%$ \\
\hline & & Clarifier Feed Launder & $0.0 \%$ \\
\hline & & Clarifier Rake & $0.1 \%$ \\
\hline
\end{tabular}

Figure 12 Model results for equipment criticality (\% downtime)

In this case, the model keeps track of the flow rate of product through a process and compares it to the expected nameplate production rate. Whenever a shortfall is noted, the model identifies the piece of equipment that is responsible for that shortfall, usually due to a failure, and tallies the duration of that shortfall. At the end of the model run, the model compiles and ranks the equipment contributing to losses in production and produces the output shown above.

These model results can be used to drive design changes and equipment sparing philosophies so that any capital expenditure would provide the greatest return on investment. It can also be used to identify equipment redundancies and potential reductions in capital cost. 


\subsection{Using model results to support decisions}

In the previous sections, we described the types of results produced by the model. The approach chosen to define the path of performance improvement to support decisions is 'the map', where we show graphically the progression of changes in model inputs on key performance metrics shown in Figure 13.

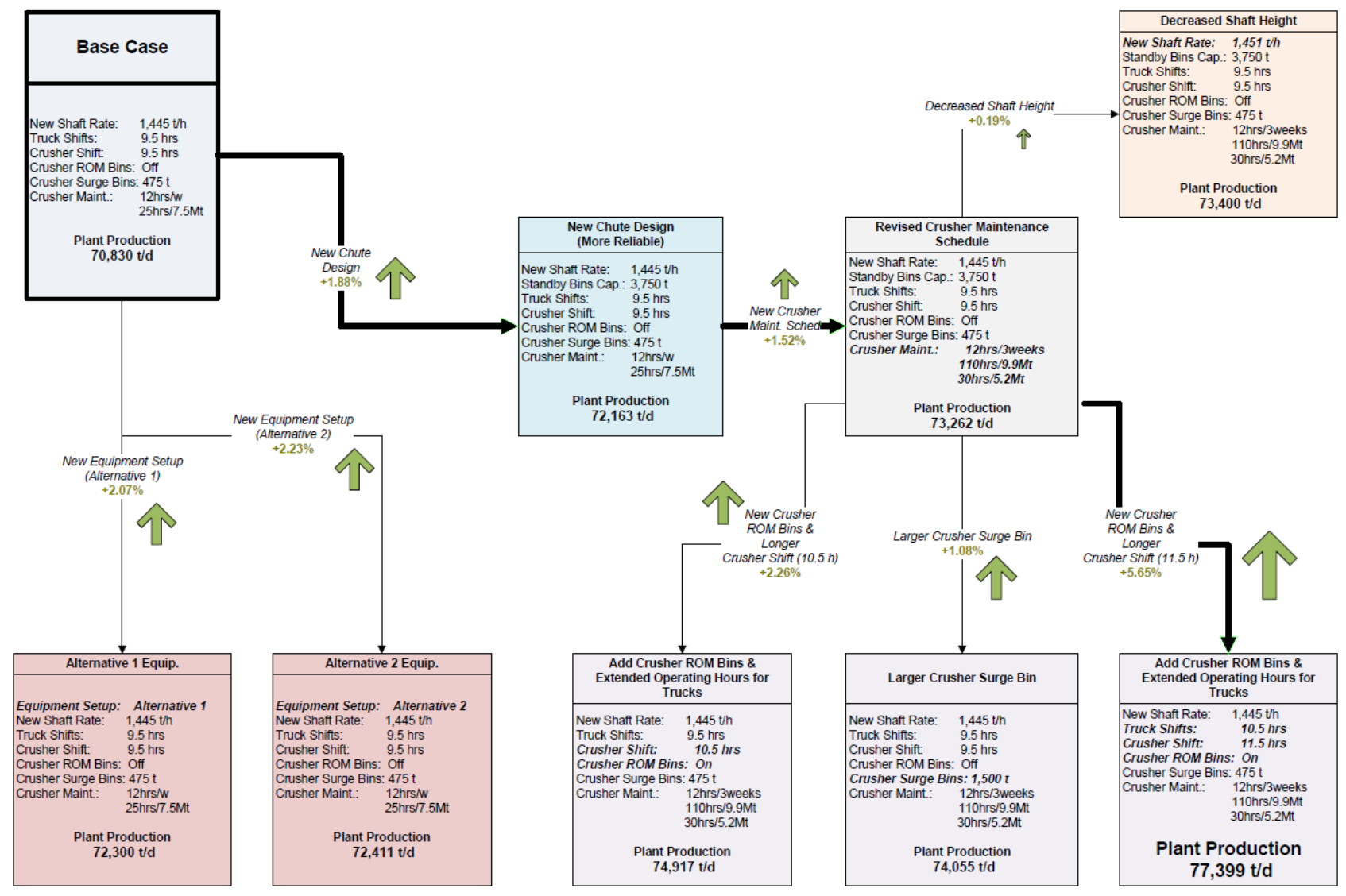

Figure 13 Example of a production map

In Figure 13 above, the base case is the starting point for the model. It is the mine design under consideration defined by process flow diagrams, design criteria, equipment lists etc. The model is built, configured and run for this base case and the key metric, Plant Production, is shown in the box in the top left $-70,830 \mathrm{t} / \mathrm{d}$.

After consultation with the client and the design team, we used the model to test the effect of possible design and operational changes that might improve performance:

- Two different equipment arrangements that altered the equipment underground.

- A different chute and gate design that was assumed to be more reliable.

- A change in the crusher maintenance schedule.

- A decrease in the height of the new shaft.

- Changes to the number of crusher and truck operating hours.

- Installation of larger crusher surge bins.

These were implemented in different combinations and the production map (Figure 13) shows the incremental change to Plant Production with each successive change such that a potential path (shown in bold) to achieving $77,399 \mathrm{t} / \mathrm{d}-$ a $9 \%$ increase in production - could be achieved with changes that were mostly operational in nature. 


\section{Software requirements and level of effort}

There are many commercial discrete-event simulation software packages that enable modelling with varying degrees of sophistication, ease-of-use, and integration with flowcharts or mine design data. Amec Foster Wheeler employ a department of engineers who specialise in building simulation models utilising Arena ${ }^{\circledR}$ simulation software because, in our opinion, it has a very open and flexible architecture allowing us to model virtually any complex system and to expand or change the model as projects develop.

To date, Amec Foster Wheeler have performed dozens of simulation studies for many clients in the mining industry. In our experience, the time and effort required to conduct a successful simulation study depends on several factors:

- The size and complexity of the design being considered.

- The level of detail to be included in the model.

- The availability of data to be used for model definition and input.

Ideally, the data provided for use as input to the study is based on historical operating data that spans several months or even years of steady-state operation. For greenfield designs or operations that do not have historical data, this data can be sourced from similar operations. Typically, we try to obtain equipment performance and availability data that includes planned and unplanned maintenance activities sufficient to develop distributions for mean times between failures and mean times to repair.

More often than not, the level of detail is commensurate with the progress of the project under study, i.e. a simulation model developed for a pre-feasibility study will be less detailed than one developed during basic engineering etc.

Although it is not possible to provide a typical cost and schedule for a simulation study without a specific scope, it is pertinent to identify that a simulation study typically takes 2 to 6 months depending on the factors listed above. Developing and examining alternative scenarios once the initial model is complete is usually much less time-intensive.

\section{Conclusion}

Underground mines are complex networks of equipment, manpower, operational considerations and logistics. Understanding how all of these factors interact with one another over time can be difficult to predict without the use of advanced tools.

A discrete-event simulation model is one of these tools.

Simulation is the process of designing a computer model of a real system based on mathematical logic. The goal is to mimic the real system with sufficient accuracy to use the model to make projections of real system behaviour under experimental conditions. Such models capture the variability inherent in complex systems and provide us with an understanding of how a system will behave under various conditions. Designing an operation to handle steady-state conditions without consideration for the peaks and valleys in day-to-day performance could result in disruptions and missed targets once the mine becomes operational.

Key reasons to simulate complex mining systems are to optimise production and to mitigate risk.

Other reasons to simulate mining systems are:

- Identify system bottlenecks.

- Quantify production rates and capacities for equipment and facilities.

- Test alternatives without shutdowns or loss of production.

- Determine system focus and strategic direction.

- Allocate resources. 
- Provide decision support for engineering.

- Develop operating procedures and philosophies.

- Train operators.

- Mitigate risk.

- Save capital.

A simulation model quantifies the risk associated with a proposed design such that potential operational extremes are identified and better-informed decisions can be made about the design, thereby improving the chances of project success.

\section{References}

Priyadarshi, H 2012, Underground mine planning, University of British Columbia, Vancouver, BC.

Quan, K \& Durston, K 2003, Dynamic simulation modelling as a decision support tool for mining design and optimization, a case study: De Beers Canada Snap Lake Diamond Mine, Canadian Institute of Mining, Metallurgy and Petroleum, Saskatoon, Canada.

Sage, AP \& Rouse, WB 2014, Handbook of systems engineering and management, 2nd edn, John Wiley \& Sons Inc., Hoboken, NJ. 